\title{
Integrative microRNA and mRNA expression profiling in acute aristolochic acid nephropathy in mice
}

\author{
ZIQIANG ZHU ${ }^{1}$, XINXING XU ${ }^{1}$, FENGYING WANG $^{2}$, YONGRUI SONG ${ }^{1}$, YANPING ZHU $^{1}$, \\ WEI QUAN ${ }^{1}$, XUELI ZHANG ${ }^{3,4}$, CHENG BI $^{3}$, HONGXIN HE$^{3},{\text { SHUANG } \text { LI }^{3} \text { and XIAOZHONG LI }}^{1}$ \\ ${ }^{1}$ Department of Nephrology and Immunology, Children's Hospital of Soochow University, Suzhou, \\ Jiangsu 215006; ${ }^{2}$ Department of Pediatrics, Sir Run Hospital, Nanjing Medical University, Nanjing, \\ Jiangsu 211100; ${ }^{3}$ Centre for Systems Biology, Soochow University, Suzhou, Jiangsu 215006, P.R. China; \\ ${ }^{4}$ School of Medicine, Institute of Medical Sciences, Örebro University, SE-70182 Örebro, Sweden
}

Received February 6, 2020; Accepted May 29, 2020

DOI: $10.3892 / \mathrm{mmr} .2020 .11444$

\begin{abstract}
In acute aristolochic acid nephropathy (AAN), aristolochic acid (AA) induces renal injury and tubulointerstitial fibrosis. However, the roles of microRNAs (miRNAs/miRs) and mRNAs involved in AAN are not clearly understood. The aim of the present study was to examine AA-induced genome-wide differentially expressed (DE) miRNAs and DE mRNAs using deep sequencing in mouse kidneys, and to analyze their regulatory networks. In the present self-controlled study, mice were treated with $5 \mathrm{mg} / \mathrm{kg} /$ day AA for 5 days, following unilateral nephrectomy. AA-induced renal injury and tubulointerstitial fibrosis were detected using hematoxylin and eosin staining and Masson's trichrome staining in the mouse kidneys. A total of 82 DE miRNAs and 4,605 DE mRNAs were identified between the AA-treated group and the self-control group. Of these DE miRNAs and mRNAs, some were validated using reverse transcription-quantitative PCR. Expression levels of the profibrotic miR-21, miR-433 and miR-132 families were significantly increased, whereas expression levels of the anti-fibrotic miR-122-5p and let-7a-1-3p were significantly decreased. Functions and signaling pathways associated with the DE miRNAs and mRNAs were analyzed using Gene Ontology and Kyoto Encyclopedia of Genes and Genomes (KEGG). A total of 767 DE pairs (in opposing directions) of miRNAs and their mRNA targets were identified. Among these, regulatory networks of miRNAs and mRNAs were analyzed using KEGG to identify enriched signaling pathways and extracellular matrix-associated pathways.
\end{abstract}

Correspondence to: Professor Xiaozhong Li, Department of Nephrology and Immunology, Children's Hospital of Soochow University, 92 Zhongnan, Suzhou Industrial Park, Suzhou, Jiangsu 215006, P.R. China

E-mail: lixiaozhongsz@163.com

Key words: integrative expression profiling, microRNA, mRNA, aristolochic acid nephropathy, regulatory network
In conclusion, the present study identified genome-wide DE miRNAs and mRNAs in the kidneys of AA-treated mice, as well as their regulatory pairs and signaling networks. The present results may improve the understanding of the role of DE miRNAs and their mRNA targets in the pathophysiology of acute AAN.

\section{Introduction}

Aristolochic acid (AA) is found in plants of the genera Aristolochia and Asarum (1). Plants of the Aristolochia genus are commonly used as herbal medicines in Asia, Central America and other countries (2). DNA damage and/or carcinogenicity is caused by AA derived-DNA adducts after AA exposure, and death or apoptosis of renal tubular cells is caused by AA-induced DNA damage $(3,4)$. AA can cause aristolochic acid nephropathy (AAN), leading to acute kidney injury and/or chronic kidney disease (CKD), accompanied by tubulointerstitial fibrosis $(5,6)$. Additionally, AA is a carcinogen associated with urothelial tumorigenesis following sufficient long-term exposure, as carcinogenicity is caused by AA derived-DNA adducts after AA exposure (6).

In acute AAN, AA induces renal injury and tubulointerstitial fibrosis (5). Fibrosis is one common final outcome of several kidney diseases (7). Renal tubulointerstitial fibrosis is a complex biological process involved in multiple cellular and signaling pathways. Moreover, fibrosis shares common pathways in several different organs (8). The progression of renal fibrosis primarily includes renal injury, recruitment and activation of immune cells, activation of fibroblasts, epithelial-to-mesenchymal transition (EMT), extracellular matrix (ECM) production and deposition, and tubular injury and atrophy (7). Pro-fibrosis factors, such as transforming growth factor- $\beta 1$ (TGF- $\beta 1$ ), are key molecular mediators of renal fibrosis $(9,10)$.

MicroRNAs (miRNAs/miRs) are a class of small, endogenous, non-coding RNAs that silence target mRNAs (11). miRNAs bind to their target mRNAs through complementary nucleotide sequences, and the target gene is suppressed by a miRNA through degradation and/or translation inhibition of its target mRNA (12). It is suggested that miRNAs 
serve a key role in renal fibrosis (13). For example, miRNAs regulate the effects of pro-fibrosis factors, EMT and ECM production (14).

To the best of our knowledge, the expression profiling of miRNAs and mRNAs, and their regulatory pairs or networks has not been reported in acute AAN. To identify the expression profiles and regulatory networks of miRNAs and mRNAs in acute AAN, the present study examined renal genome-wide differentially expressed (DE) miRNAs and DE mRNAs using deep sequencing in mouse kidneys induced by a short-term exposure to AA.

\section{Materials and methods}

The flow chart of the experimental design of the present study is shown in Fig. 1.

Animal treatment. A total of four kidney samples were used as the self-control group, and were obtained through single nephrectomy from four 8-week-old male C57BL/6 mice (specific pathogen free; weight, $\sim 20 \mathrm{~g}$; Experimental Animal Center of Soochow University). The mice were raised in a specific pathogen free animal house with a constant temperature $\left(24^{\circ} \mathrm{C}\right)$, humidity $(50 \%)$, specific pathogen free filtered atmosphere and $12 \mathrm{~h} \mathrm{light/dark} \mathrm{cycles.} \mathrm{All} \mathrm{the} \mathrm{mice} \mathrm{had} \mathrm{free}$ access and were fed with radiation sterilization food and water sterilized with high temperature and high pressure. The mice were anesthetized using chloral hydrate $(400 \mathrm{mg} / \mathrm{kg}$ intraperitoneal injection) before single nephrectomy. After 4 weeks, these four mice were treated with an intraperitoneal injection of AA I (cat. no. A5512; Sigma-Aldrich; Merck KGaA) at a dosage of $5 \mathrm{mg} / \mathrm{kg} / \mathrm{day}$ for 5 days. A total of $100 \mathrm{mg}$ AA I was diluted with $5 \mathrm{ml}$ DMSO (final concentration, 5\%) and $95 \mathrm{ml}$ saline. The final concentration of the injected AA I was $1 \mathrm{mg} / \mathrm{ml}$. All mice were euthanized by cervical dislocation. The death of mice was verified by the stopping of both breathing and the heartbeat. The remaining four kidney samples were obtained when these four mice were sacrificed on the 6th day. All kidney samples were divided into two parts, one of which was fixed in $10 \%$ formalin solution for 5 days at room temperature, while the other was frozen at $-80^{\circ} \mathrm{C}$ in TRIzol $^{\circledR}$ reagent (Invitrogen; Thermo Fisher Scientific, Inc.). The present study was approved by the Ethics Committee of the Children's Hospital of Soochow University (Suzhou, China; approval no. 2020-CHSU-012).

Histological analysis. After fixing in 10\% formalin solution for $\geq 3$ days at $26^{\circ} \mathrm{C}$, all kidney samples were dehydrated and transparent as follows: $70 \%$ alcohol for $1 \mathrm{~h}, 80 \%$ ethanol for $1 \mathrm{~h}, 90 \%$ ethanol for $1 \mathrm{~h}, 95 \%$ ethanol for $1 \mathrm{~h}$, anhydrous ethanol twice for $1 \mathrm{~h}$, anhydrous ethanol and xylene solution (1:1) for $1 \mathrm{~h}$ and xylene twice for $1 \mathrm{~h}$. The kidney samples were embedded in paraffin and sectioned into 5 - $\mu \mathrm{m}$-thick slices. The slices were placed in at $60^{\circ} \mathrm{C}$ for $30 \mathrm{~min}$, dewaxed after being immersed in xylene twice for $10 \mathrm{~min}$ each time and then immersed in ethanol $(100,95,80$ and $70 \%)$ and deionized water for $5 \mathrm{~min}$. Sections of the kidney samples were stained with hematoxylin and eosin (HE) or Masson's trichrome. HE staining were performed as follow: Hematoxylin dye for $10 \mathrm{~min}$ at $26^{\circ} \mathrm{C}$, rinsed with water and $0.7 \%$ hydrochloric acid ethanol for $5 \mathrm{sec}$. Cells were then washed with water until the cell nuclei were blue, along with $95 \%$ ethanol for $30 \mathrm{sec}$, alcoholic eosin dye for $30 \mathrm{sec}$ at $26^{\circ} \mathrm{C}, 95 \%$ ethanol twice for $30 \mathrm{sec}, 100 \%$ ethanol twice for $5 \mathrm{~min}$, carbolic Acid and xylene (1:4) for $5 \mathrm{~min}$, xylene twice for $5 \mathrm{~min}$ and the slices were sealed with neutral balsam.

Masson's trichrome staining was performed as follow: Staining with Regaud's hematoxylin dye for $10 \mathrm{~min}$ at $26^{\circ} \mathrm{C}$, rinsed with unstained color with water, $0.7 \%$ hydrochloric acid ethanol for $5 \mathrm{sec}$, washed with water for 8 min, ponceaux and acid fuchsin $(0.7 \%$ ponceaux, $0.3 \%$ acid fuchsin and $1 \%$ acetic acid) for $8 \mathrm{~min}, 2 \%$ acetic acid solution for $45 \mathrm{sec}$, $1 \%$ phosphomolybdic acid for $4 \mathrm{~min}$, aniline blue for $5 \mathrm{~min}$ and $0.2 \%$ acetic acid until no obvious blue color escaped from the slices. Subsequently, slices were rinsed with $95 \%$ ethanol twice for $30 \mathrm{sec}, 100 \%$ ethanol twice for $5 \mathrm{~min}$ and xylene twice for $5 \mathrm{~min}$, and then sealed with neutral balsam. The slices were observed and captured at $\times 400$ magnification under a light microscope.

RNA extraction. Total RNA was extracted from the kidney samples using TRIzol ${ }^{\circledR}$ reagent according to the manufacturer's protocol. Total RNA was qualified and quantified using NanoDrop and an Agilent 2100 bioanalyzer (both from Thermo Fisher Scientific, Inc.).

Sequencing of miRNAs. Small RNAs from the total RNA were obtained from the 18-30 nt region following 15\% urea polyacrylamide gel electrophoresis (urea-PAGE) gel electrophoresis. After the addition of 3' and 5' adaptors (MGIEasy Small RNA Library Prep Kit V2.0; BGI Genomics), the cDNA of small RNAs was synthesized and enriched by reverse transcription and PCR amplification. The reverse transcription was performed using the First Strand Master Mix and Superscript II Reverse Transcriptase (SuperScript ${ }^{\mathrm{TM}}$ First-Strand Synthesis System; Invitrogen; Thermo Fisher Scientific, Inc.) at $42^{\circ} \mathrm{C}$ for $1 \mathrm{~h}$ and $70^{\circ} \mathrm{C}$ for $15 \mathrm{~min}$. PCR was performed using PCR Primer Cocktail and PCR Master Mix (MGIEasy Small RNA Library Prep Kit V2.0; BGI Genomics) to enrich the cDNA fragments, under the following conditions: Initial denaturation at $95^{\circ} \mathrm{C}$ for $3 \mathrm{~min}$, followed by 18 cycles at $98^{\circ} \mathrm{C}$ for $20 \mathrm{sec}, 56^{\circ} \mathrm{C}$ for $15 \mathrm{sec}$ and $72^{\circ} \mathrm{C}$ for $15 \mathrm{sec}$, with a final extension at $72^{\circ} \mathrm{C}$ for $10 \mathrm{~min}$. The library of small RNAs was constructed from the PCR products in the 100-120 bp region, separated by agarose gel electrophoresis, eliminating primer-dimers and other potential byproducts. The library of small RNAs was denatured by heat $\left(94^{\circ} \mathrm{C}\right)$, and the single-stranded DNA was cyclized. The library of cyclized small RNAs was sequenced using a BGISEQ-500 platform (BGI Genomics). The clean data was filtered from the raw data. The clean reads of miRNAs were mapped to the mouse genome and other sRNAs database with annotation using the software Anchor Alignment-Based Small RNA Annotation version 1.0 (University of Nevada) (15), except Rfam version 12.0, which was performed using cmsearch version 1.1 (16).

Sequencing of mRNAs. mRNA was selected using poly-T oligo-attached magnetic beads, and ribosomal RNA was depleted. mRNA was fragmented and reverse transcribed into double-stranded cDNA using N6 random primers. 


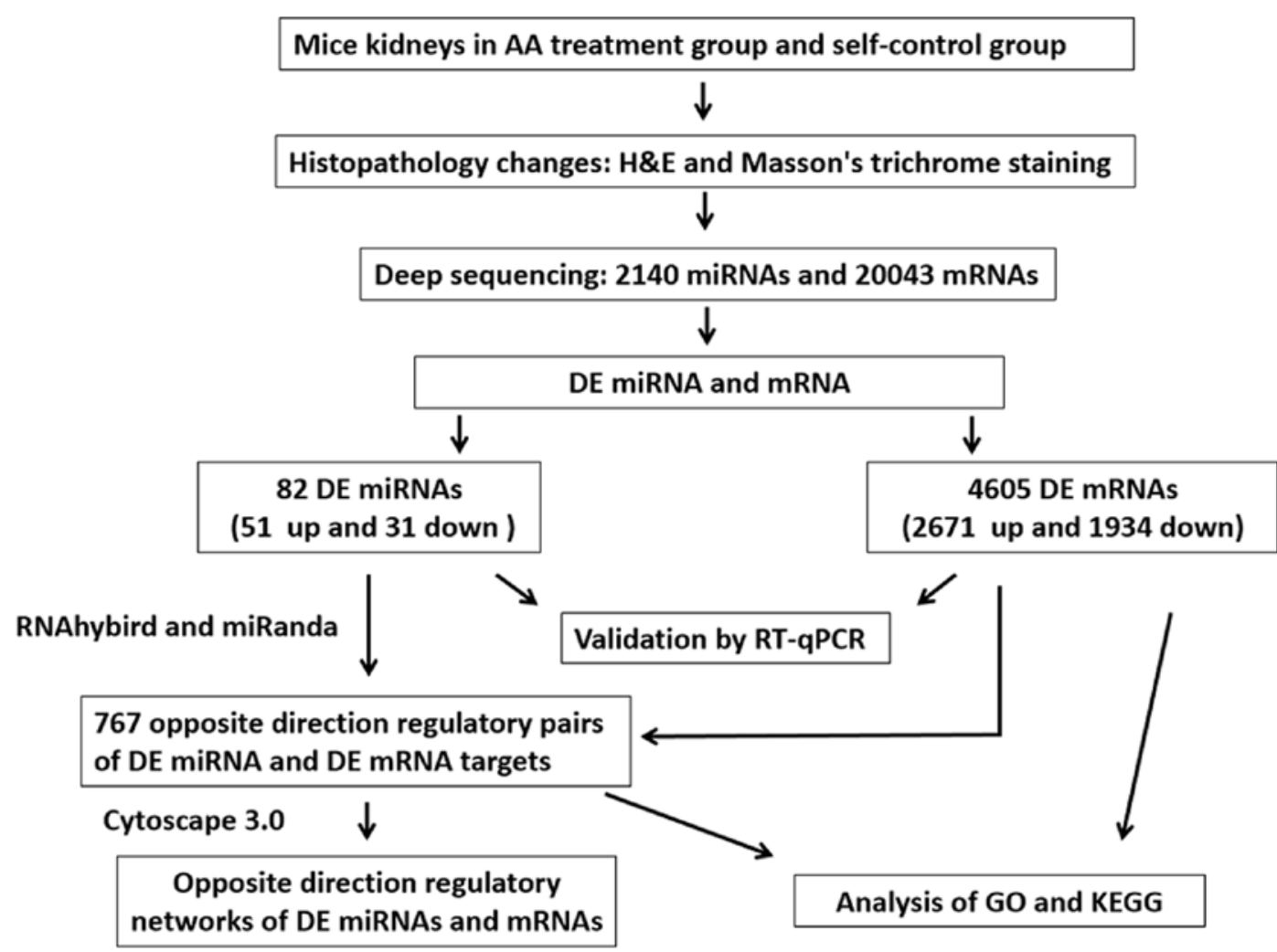

Figure 1. Flow chart of the experimental design. AA, aristolochic acid; H\&E, hematoxylin and eosin; miRNA, microRNA; DE, differentially expressed; GO, Gene Ontology; KEGG, Kyoto Encyclopedia of Genes and Genomes.

The reverse transcription was performed using the First Strand Master Mix, N6 random primers and Superscript II Reverse Transcriptase (SuperScript ${ }^{\mathrm{TM}}$ First-Strand Synthesis System; Invitrogen; Thermo Fisher Scientific, Inc.) at $42^{\circ} \mathrm{C}$ for $1 \mathrm{~h}$ and $70^{\circ} \mathrm{C}$ for $15 \mathrm{~min}$. The dscDNA was subjected to end-repair and 3'-adenylation. A bubble adapter was ligated to the double ends of the dscDNA. After purifying, the ligated products were amplified by PCR. PCR was performed using PCR Primer Cocktail and PCR Master Mix (MGIEasy RNA Library Prep Kit V3.0; BGI Genomics) to enrich the cDNA fragments, under the following conditions: Initial denaturation at $95^{\circ} \mathrm{C}$ for $3 \mathrm{~min}$, followed by 18 cycles at $98^{\circ} \mathrm{C}$ for $20 \mathrm{sec}$, $56^{\circ} \mathrm{C}$ for $15 \mathrm{sec}$ and $72^{\circ} \mathrm{C}$ for $15 \mathrm{sec}$; with a final extension at $72^{\circ} \mathrm{C}$ for $10 \mathrm{~min}$. The PCR products were denatured by heat $\left(94^{\circ} \mathrm{C}\right)$ and the single-stranded DNA was cyclized using splint oligo and DNA ligase. The cyclized mRNA was sequenced using the BGISEQ-500 platform. The clean mRNA data were filtered from the raw data and mapped to the mouse genome with annotation using HISAT version 2.0.4 (Center for Computational Biology; Johns Hopkins University) (17). A detailed explanation of the methods of miRNA and mRNA sequencing and analysis is provided in Data $\mathrm{S} 1$.

Functional analysis of the DE miRNAs and DE mRNAs. Hierarchical clustering analysis was performed between all 8 kidney samples using function hclust of $\mathrm{R}$ software version 3.5.2 (R Core Team). Principal component analysis (PCA) was performed with all 8 kidney samples using function princomp of $\mathrm{R}$ software version 3.5.2 (R Core Team). Between the AA-treated group and the self-control group, DE miRNAs were defined using a cut-off with read count $>50$, adjusted P-values $<0.001$ and $\log _{2}$ fold changes (AA/control) $>1.5$ or $<-1.5$. DE mRNAs were defined using a cut-off with read count $>50$, adjusted $\mathrm{P}$-values $<0.001$ and $\log _{2}$ fold changes (AA/control) $>1.0$ or $<-1.0$. These cut-off points were used to assist in identifying the key DE miRNAs and DE mRNAs enriched in biological processes and pathways, which may be involved in the pathophysiology of acute AAN.

Target genes of miRNAs were identified based on the similarity of the sequences with target genes, predicted using RNAhybrid version 2.2 (18) and miRanda (version released in 2010) (19). The functions and pathways analysis of both DE mRNAs and the target genes of DE miRNAs were performed using Gene Ontology (GO) (20) and the Kyoto Encyclopedia of Genes and Genomes (KEGG) databases (21). Networks of DE miRNAs and DE mRNAs were drawn using Cytoscape version 3.0 (National Institute of General Medical Sciences).

Reverse transcription-quantitative $(R T-q) P C R$. To validate the DE miRNA and DE mRNA results, RT-qPCR was performed on reference DE miRNAs and DE mRNAs from the total RNA of all 8 kidney samples extracted as aforementioned. RT-qPCR assays were performed using a Light Cycler 480 II real-time PCR system (Roche Diagnostics). U6 and GAPDH were used as the internal controls for miRNA and mRNA, respectively. The M-MLV Reverse Transcriptase kit (Promega Corp.) was used for the synthesis of cDNA, using the following conditions: $42^{\circ} \mathrm{C}$ for $1 \mathrm{~h}$ and $70^{\circ} \mathrm{C}$ for $10 \mathrm{~min}$. The cDNA products of mRNA were amplified using SYBR 
Premix Ex Taq kit (Takara Bio, Inc.) using the following thermocycling conditions: $95^{\circ} \mathrm{C}$ for $30 \mathrm{sec}$; followed by 40 cycles of $95^{\circ} \mathrm{C}$ for $5 \mathrm{sec}$ and $60^{\circ} \mathrm{C}$ for $30 \mathrm{sec}$, and a final cycle of $95^{\circ} \mathrm{C}$ for $15 \mathrm{sec}, 60^{\circ} \mathrm{C}$ for $30 \mathrm{sec}$ and $95^{\circ} \mathrm{C}$ for $15 \mathrm{sec}$. All the primers (Shanghai GeneChem Co., Ltd.) for detection of mRNAs are listed in Table I. The RT-qPCR reactions for all miRNAs were performed using a miDETECTA Track ${ }^{\mathrm{TM}}$ miRNA qRT-PCR Starter kit (Guangzhou RiboBio Co., Ltd.) according to the manufacturer's protocol. RT-qPCR was performed in triplicate. The expression levels of the selected miRNAs and mRNAs were normalized to those of U6 and GAPDH, respectively. The changes in expression of the assessed miRNAs and mRNAs between the AA treatment group and the self-control group were calculated using the $2^{-\Delta \Delta \mathrm{Cq}}$ method (22).

Statistical analysis. Data are presented as the mean \pm standard error of the mean. Statistical analysis was performed using GraphPad Prism version 8.0 (GraphPad Software, Inc.). Statistical differences were calculated using a paired Student's t-test. $\mathrm{P}<0.05$ was considered to indicate a statistically significant difference.

\section{Results}

Renal histopathological alterations induced by AA. Histopathological changes in the kidney samples were detected using H\&E and Masson's trichrome staining. There were markedly widened tubular lumens and flattened tubular cells in the AA-treated group compared with in the self-control group (Fig. 2A-D). Tubulointerstitial fibrosis was induced by AA treatment, as shown by the observable increase in collagen based on the Masson's trichrome staining in the AA-treated group compared with in the self-control group (Fig. 2C and D).

Renal DE mRNAs induced by AA. A total of 20,043 mRNAs were detected using deep sequencing in the 8 kidney samples. The mRNA expression patterns were significantly different between the AA-treated group and the self-control group based on hierarchical clustering analysis and PCA (Fig. 3).

Among the total detected mRNAs, 4,605 were considered to be significantly DE between the AA-treated group and the self-control group. Of these, 2,671 DE mRNAs were upregulated and 1,934 were downregulated.

AA treatment induced extensive alterations in cellular function and processes based on the results of GO and KEGG pathway analysis on DE mRNAs. GO analysis of DE mRNAs identified 'single-organism process' and 'cellular process' as the most altered biological process, 'cell' and 'cell part' as the most altered cellular component, and 'binding' and 'catalytic activity' as the most altered molecular function (Fig. 4A). KEGG analysis identified 'signal transduction', 'global and overview maps', 'cancers: Overview', 'immune system' and 'endocrine system' as the most altered pathways (Fig. 4B). Furthermore, 'amino acid metabolism', 'fatty acid degradation', 'complement and coagulation cascades' and 'ECM-receptor interaction' were the most enriched pathways (Fig. 4C). The 'amino acid metabolism' included all types of amino acid metabolism, such as 'Arginine and proline metabolism', 'Alanine, Aspartic acid and glutamate metabolism', 'glycine, serine and threonine metabolism' and 'Valine, leucine and isoleucine degradation'.

RenalDEmiRNAs induced by AA.A total of 2,140miRNAs were detected by deep sequencing. Among these, 82 DE miRNAs were identified between the AA-treated and the self-control group (Table SI). Of these, 51 DE miRNAs were upregulated in the AA treatment group compared with in the self-control group, and $31 \mathrm{DE}$ miRNAs were downregulated.

The top 10 upregulated miRNAs in this study were miR-124-5p, miR-122-3p, miR-124-3p, miR-344d-3p, miR-409-5p, miR-129-2-3p, miR-496a-3p, miR-147-3p, miR-5128 and miR-7649-3p (Table SI). Among the 51 upregulated DE miRNAs, miRNAs with the highest expression levels in the AA treatment group were miR-21a-5p, miR-31-5p, miR-21a-3p, miR-146b-5p, miR-212-3p, miR-34b-3p, miR-132-3p, miR-34c-3p and miR-34c-5p. The 10 most downregulated miRNAs were miR-3073a-5p, miR-3073b-3p, miR-3073b-5p, miR-1948-5p, miR-92a-2-5p, miR-3073a-3p, miR-669c-5p, miR-7085-5p, miR-1968-5p and miR-1948-3p (Table SI). Among the 31 downregulated DE miRNAs, the highest expressed miRNAs in the self-control group were miR-187-3p, miR-190a-5p, miR-122-5p, miR-486a-5p and let-7a-1-3p.

The profibrotic miR-21, miR-433 and miR-132 families, and the oncogenic miR-34 family were significantly increased, while the anti-fibrotic miR-122-5p and let-7a-1-3p were significantly decreased following AA treatment.

Validation of DE miRNAs and DE mRNAs. To validate the DE miRNAs and DE mRNAs, RT-qPCR was performed on miR-21a-5p, miR-324-3p, miR-132-3p, miR-1968-5p, TIMP metallopeptidase inhibitor 1 (Timp1), serpin family $\mathrm{E}$ member 1 (Serpine1), interleukin (IL)-11 and TGF- $\beta 1$. These miRNA and mRNA were chosen as they were among the most altered mRNAs and miRNAs, which also had high expression levels (high read counts). Moreover, the chosen mRNAs are pro-fibrosis factors $(23,24)$. As shown in Fig. 5, changes in expression levels of the assessed miRNAs and mRNAs based on RT-qPCR were consistent with the results of deep sequencing (Table SI). Relative expression levels of miR-21a-5p (3.90 \pm 0.90 vs. $1.00 \pm 0.08)$, miR-324-3p (2.87 \pm 0.62 vs. $1.00 \pm 0.13)$ and miR-132-3p (3.81 \pm 0.77 vs. $1.00 \pm 0.25)$ were significantly increased in the AA-treated group compared with that in the self-control group, respectively, and the expression level of miR-1968-5p (0.23 \pm 0.02 vs. $1.00 \pm 0.07)$ was significantly decreased (Fig. 5A-D). Relative mRNA levels of Timp1 (304.24 \pm 88.65 vs. $1.00 \pm 0.56)$, Serpinel (92.07 \pm 44.17 vs. $1.00 \pm 0.56)$, IL-11 (23.25 \pm 1.48 vs. $1.00 \pm 0.47)$ and transforming growth factor (TGF)- $\beta 1$ (3.85 \pm 0.62 vs. $1.00 \pm 0.16)$ were significantly increased in the AA-treated group compared with that in the self-control group (Fig. 5E-H).

Integrated analysis of DE miRNA-DE mRNA target pairs. Targets of DE miRNAs were predicted using both RNAhybrid and miRanda. A total of 8,230 pairs of DE miRNA-mRNA targets were found. Compared with the DE mRNA data in the present study, 1,569 pairs of DE miRNA-DE mRNA targets were found. Among these, there was a total of 767 opposite direction regulatory pairs of DE miRNA-DE mRNA targets, 
Table I. Sequences of primers used for reverse transcription-quantitative PCR.

\begin{tabular}{lll}
\hline mRNAs & \multicolumn{1}{c}{ Forward $\left(5^{\prime}-3^{\prime}\right)$} & \multicolumn{1}{c}{ Reverse $\left(3^{\prime}-5^{\prime}\right)$} \\
\hline GAPDH & TGGTGAAGGTCGGTGTGAAC & GCTCCTGGAAGATGGTGATGG \\
TGF- $\beta 1$ & AGCCTGCCTCTTGAGTCCCT & CTCCCAAGGAAAGGTAGGTGAT \\
IL-11 & GCTGGGACATTGGGATCTTTG & GAGCTGTAAACGGCGGAGTAG \\
Timp1 & CCCCAGAAATCAACGAGACC & GTACGCCAGGGAACCAAGAA \\
Serpine1 & GACACCCTCAGCATGTTCATC & TTGGTCGGAAAGACTTGTGAA \\
U6 & CTCGCTTCGGCAGCACA & AACGCTTCACGAATTTGCGT \\
miR-21a-5p & GUACUUAUCAGACUGAUGUUGA \\
miR-124-3p & TCTTTAAGGCACGCGGTG & UCAACAUCAGUCUGAUAAGUUA \\
miR-132-3p & GCGCGCGTAACAGTCTACAGG & TATGGTTTTGACGACTGTGTGAT \\
miR-1968-5P & TGCAGCTGTTAAGGATGGTGGACT & GTCGTATCCAGTGCAGGGTCC \\
\end{tabular}

IL-11, interleukin 11; TGF- $\beta 1$, transforming growth factor $\beta 1$; miR, microRNA; Timp1, TIMP metallopeptidase inhibitor 1; Serpine1, serpin family E member 1 .
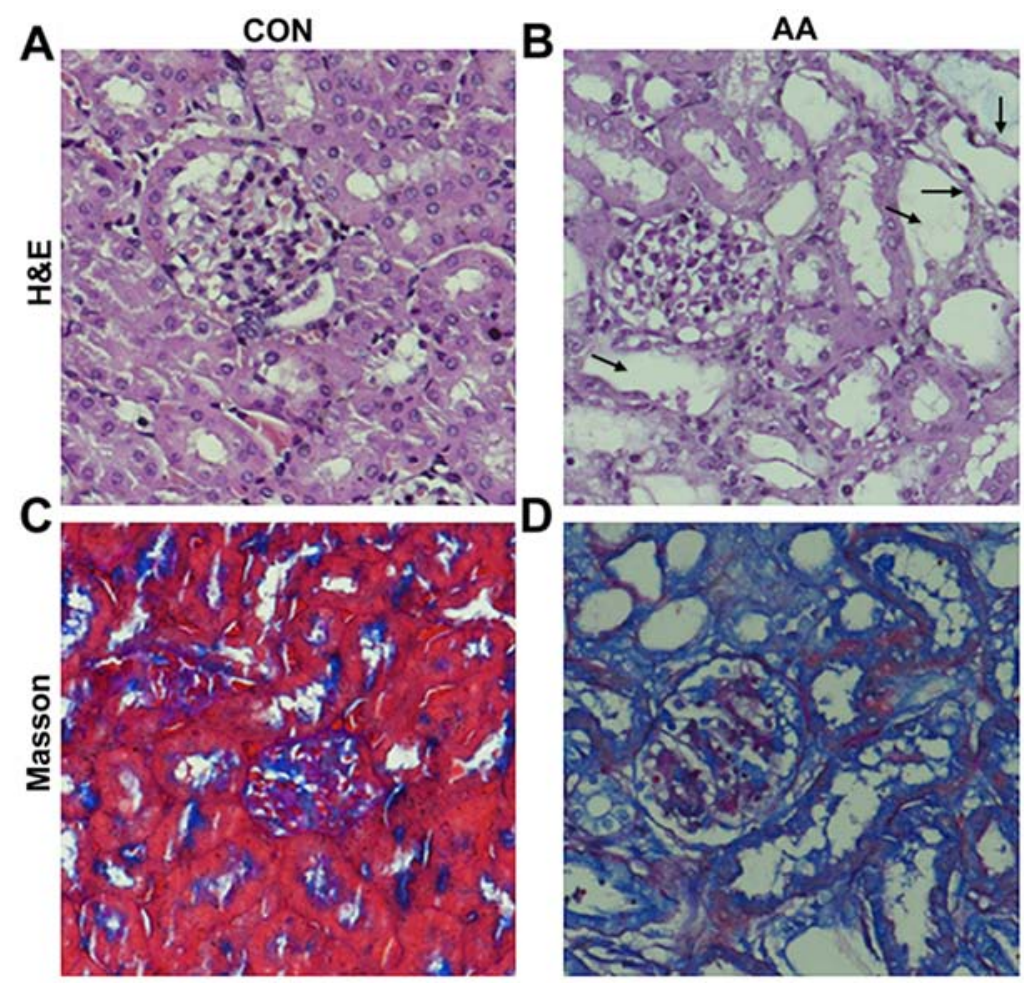

Figure 2. Histopathological changes induced by AA treatment analyzed by H\&E and Masson's trichrome staining in kidney samples. Magnification, $\mathrm{x} 400$. Compared with (A) the self-control group, notably widened tubular lumens and flattened tubular cells were observed in (B) the AA-treated group by H\&E staining. The arrows pointed out to the representative widened tubular lumens or flattened tubular cells Compared with (C) the self-control group, a notable increase in collagen, as shown by an increase in Masson's trichrome staining, was observed in (D) the AA-treated group. AA, aristolochic acid; H\&E, hematoxylin and eosin; $\mathrm{CON}$, control.

including 416 pairs of upregulated DE miRNAs and downregulated DE mRNAs, and 351 pairs of downregulated DE miRNAs and upregulated DE mRNAs (Data S2).

These 767 opposite direction regulatory pairs of DE miRNAs and DE mRNAs included 82 DE miRNAs and 624 DE mRNAs. Using GO analysis on the 624 DE mRNAs, it was identified that 'cellular process', 'biological regulation' and 'regulation of biological process' were the most notably altered biological processes, 'binding' was the most changed molecular function, and 'cell' and 'cell part' were the most highly altered cellular components (Fig. 6A).

KEGG analysis on the aforementioned 624 DE mRNAs identified 'signal transduction', 'global and overview maps', 'cancers', 'endocrine system', 'cellular community-eukaryotes' and 'immune system' as the main altered pathways (Fig. 6B). The results were similar to that of total DE mRNAs. The most enriched KEGG pathways were identified as signaling pathways, ECM-associated pathways, 'metabolic pathways' and 'pathways 
A

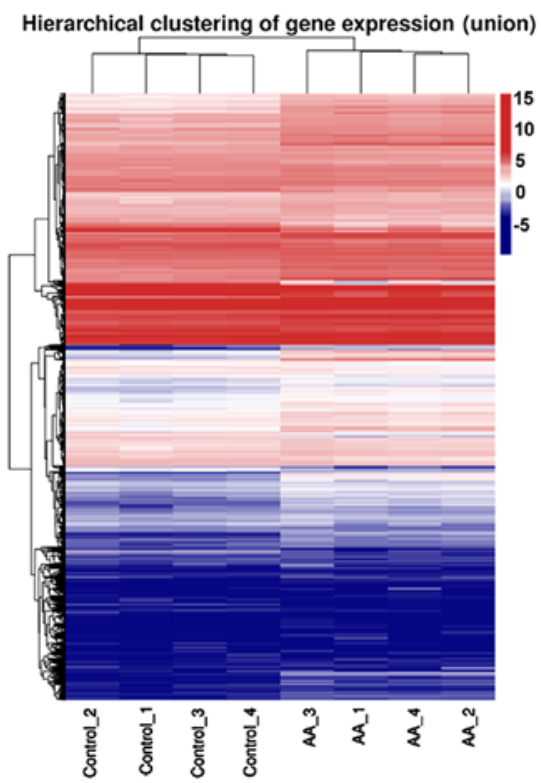

B

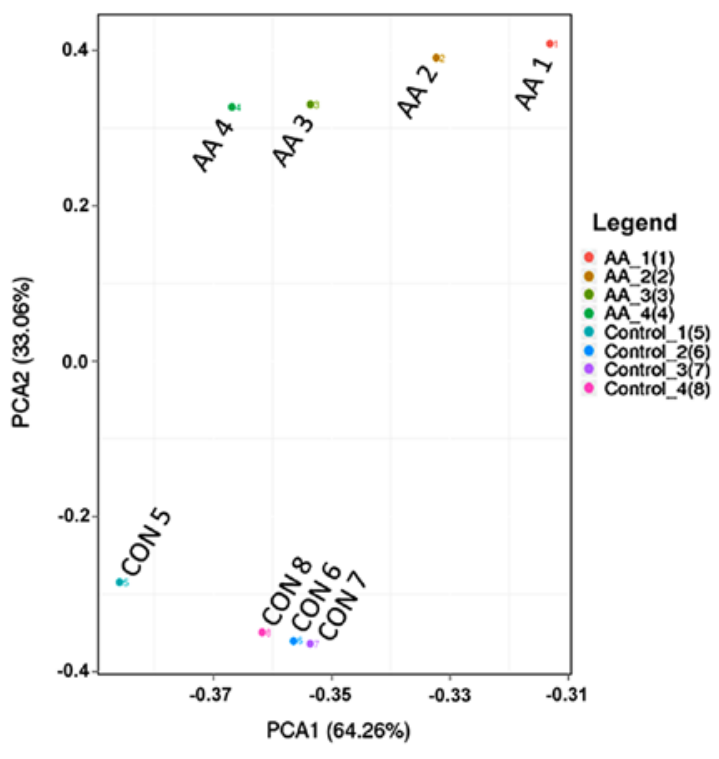

Figure 3. HCA and PCA of mRNA expression patterns. HCA and PCA were used to separate all 8 samples into different groups or clusters. Each group or cluster had similar mRNA expression patterns. (A) All 8 samples were separated into two groups, consistent with the treatment groups and the self-control groups, by HCA of mRNA expression levels. Each column represents an individual sample. Each row represents a differentially expressed mRNA. Different colors represent different expression levels of mRNA. The intensity of the red color is associated with higher expression levels. By contrast, the intensity of the blue color is associated with lower expression levels. (B) PCA separated all 8 samples into the AA-treated and the self-control clusters. PCA decreased a large amount of mRNA expression information in samples to a few independent variables (principal components) to compare the samples and identify clusters with high similarities in mRNA expression levels. The separate points represent separate samples. The $\mathrm{x}$ - and y-axes represent the contributor rate of the first component and second component, respectively. HCA, hierarchical clustering analysis; PCA, principal component analysis; AA, aristolochic acid.

A

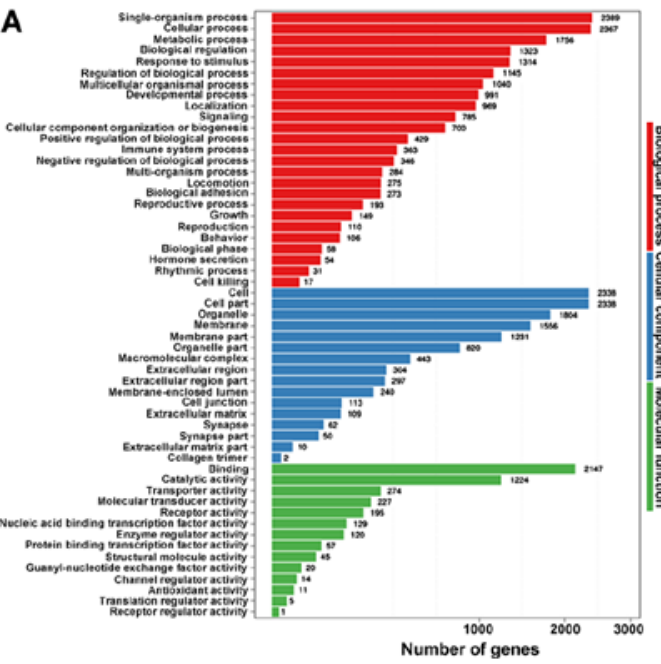

B
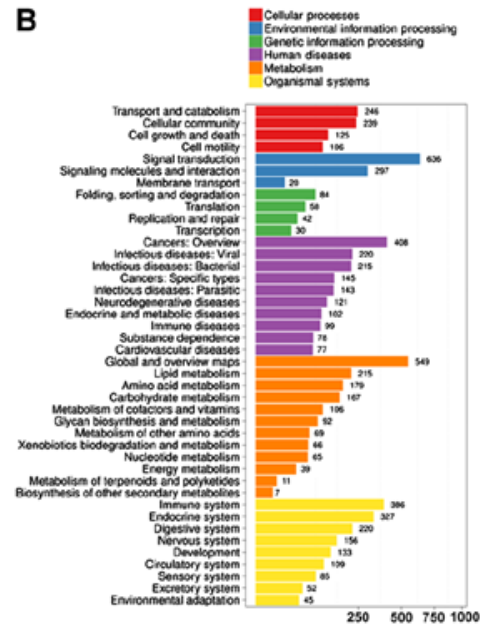

Number of genes
C

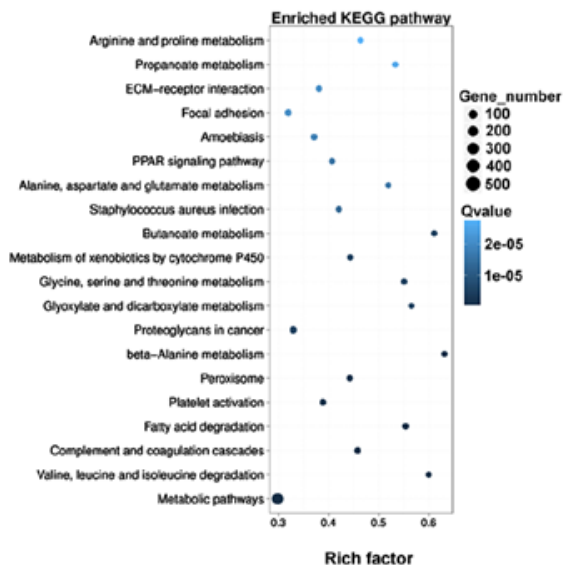

Figure 4. Analysis of GO and KEGG pathways of differentially expressed mRNAs. (A) GO analysis. (B) KEGG analysis of pathway enrichment. (C) Enriched KEGG pathways. GO, Gene Ontology; KEGG, Kyoto Encyclopedia of Genes and Genomes.

in cancer' (Fig. 6C). Signal transduction was the most changed pathway, and signaling pathways were the most enriched pathways. A total of 40 signaling pathways and 6 ECM-associated pathways were identified by KEGG analysis. The most altered signaling pathways included 'Hedgehog signaling pathway', 'ABC transporters', 'ErbB signaling pathway', 'cAMP signaling pathway', 'Relaxin signaling pathway', 'Ras signaling pathway' and 'PI3K-Akt signaling pathway' (Fig. 6C). The most altered ECM-associated pathways included 'ECM-receptor interaction' and 'focal adhesion' (Fig. 6C).
Regulatory networks of DE miRNAs on DE mRNAs involved in signaling pathways and ECM-associated pathways following AA treatment. Signaling pathways were the most enriched KEGG pathways. Additionally, ECM-associated pathways were enriched pathways and closely associated with renal fibrosis. A total of 63 DE miRNAs and their 107 DE mRNA targets were found from the aforementioned 40 signaling pathways and 6 ECM-associated pathways. As shown in Fig. 7, opposing direction regulatory networks of DE miRNAs-DE mRNAs included 134 DE miRNA-DE mRNA pairs (Table SII). Regulatory 

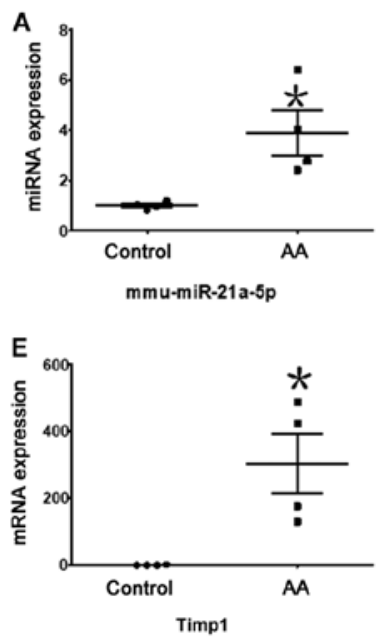
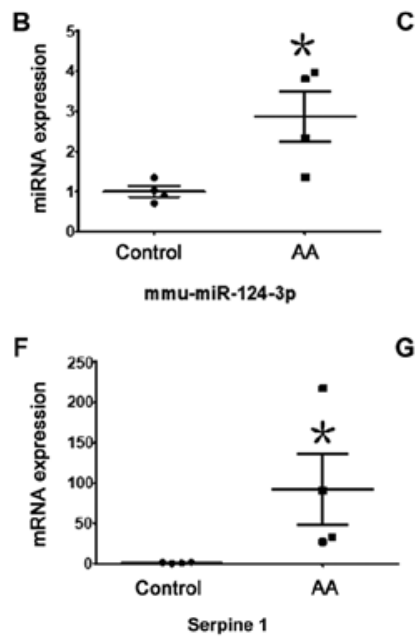

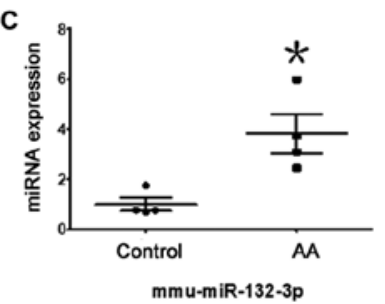

G

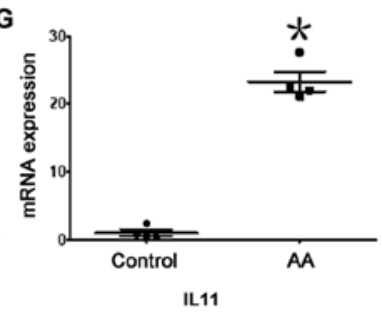

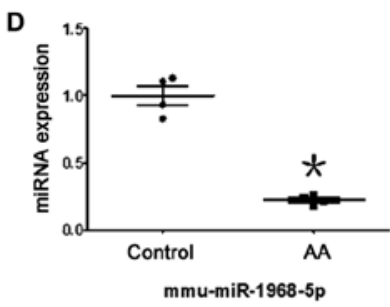

H

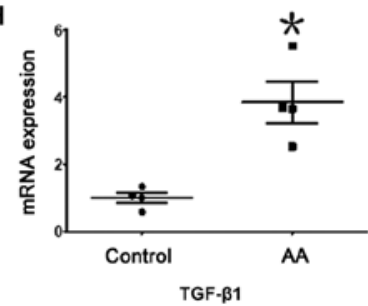

Figure 5. Expression levels of DE miRNAs and DE mRNAs validated by RT-qPCR. Expression levels of 4 DE miRNAs and 4 DE mRNAs were measured using RT-qPCR and normalized to U6 or GAPDH, respectively. Relative expression levels of (A) miR-124-3p ( $P=0.0398),(B) m i R-21 a-5 p(P=0.0391)$ and (C) miR-132-3p ( $\mathrm{P}=0.0399)$ were significantly increased in the AA group compared with in the self-control group. Expression levels of (D) miR-1968-5p $(\mathrm{P}=0.0014)$ were significantly decreased in the AA group compared with in the self-control group. Relative mRNA levels of $(\mathrm{E})$ Timp1 $(\mathrm{P}=0.0416),(\mathrm{F}) \mathrm{Serpine1}$ $(\mathrm{P}=0.0475),(\mathrm{G})$ IL11 $(\mathrm{P}=0.0038)$ and $(\mathrm{H})$ TGF- $\beta 1(\mathrm{P}=0.0146)$ were significantly increased in the AA group compared with in the self-control group. " $\mathrm{P}<0.05$. AA, aristolochic acid; DE, differentially expressed; miRNA/miR, microRNA; RT-qPCR, reverse transcription-quantitative PCR; AA, aristolochic acid; IL11, interleukin 11 ; TGF- $\beta 1$, transforming growth factor $\beta 1$; mmu, Mus musculus.

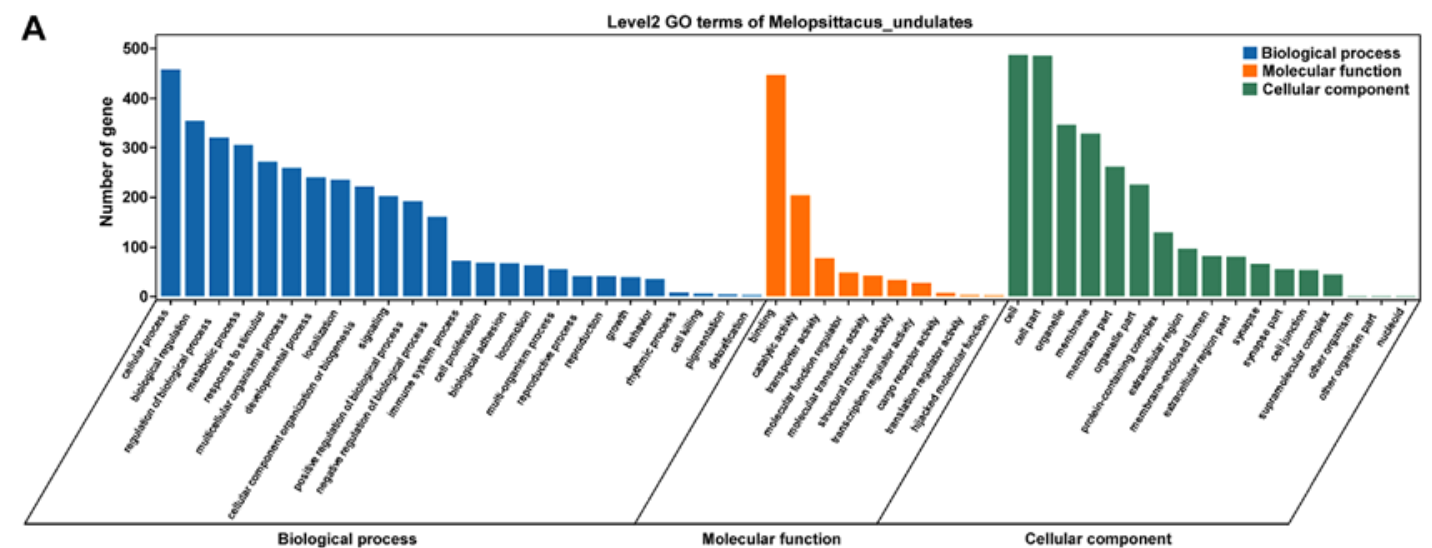

B

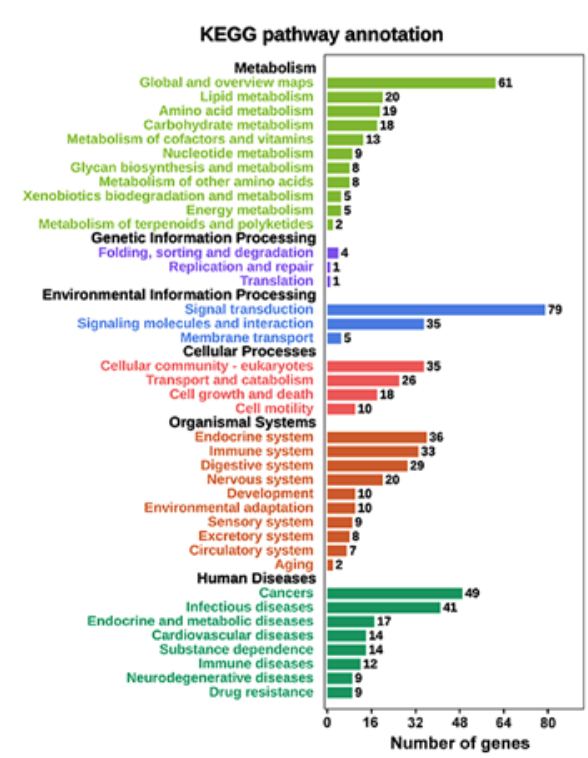

C

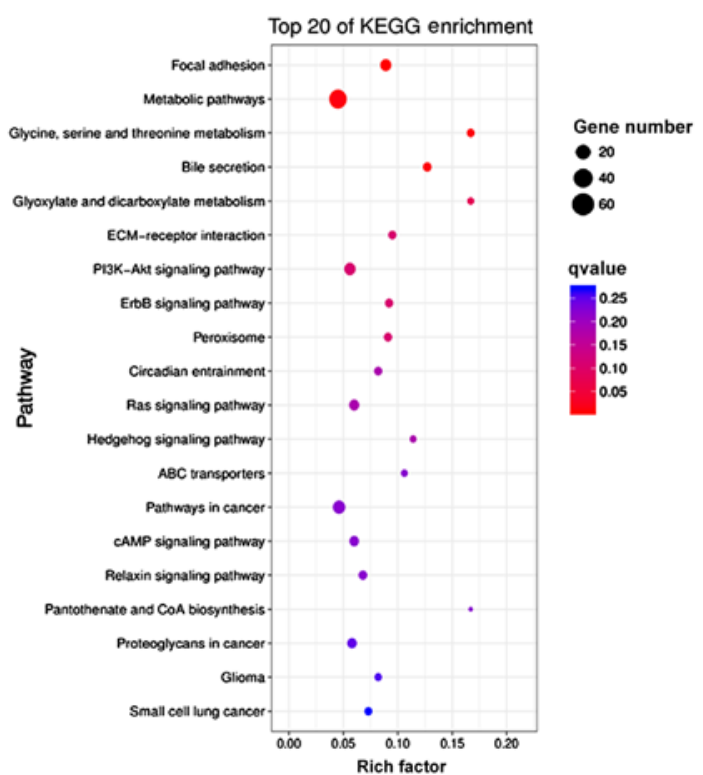

Figure 6. GO and KEGG pathway analysis of DE miRNAs and concurrently oppositely regulated DE mRNAs. (A) GO analysis. (B) KEGG analysis of pathway enrichment. (C) Enriched KEGG pathways. GO, Gene Ontology; KEGG, Kyoto Encyclopedia of Genes and Genomes; DE, differentially expressed; miRNA, microRNA. 


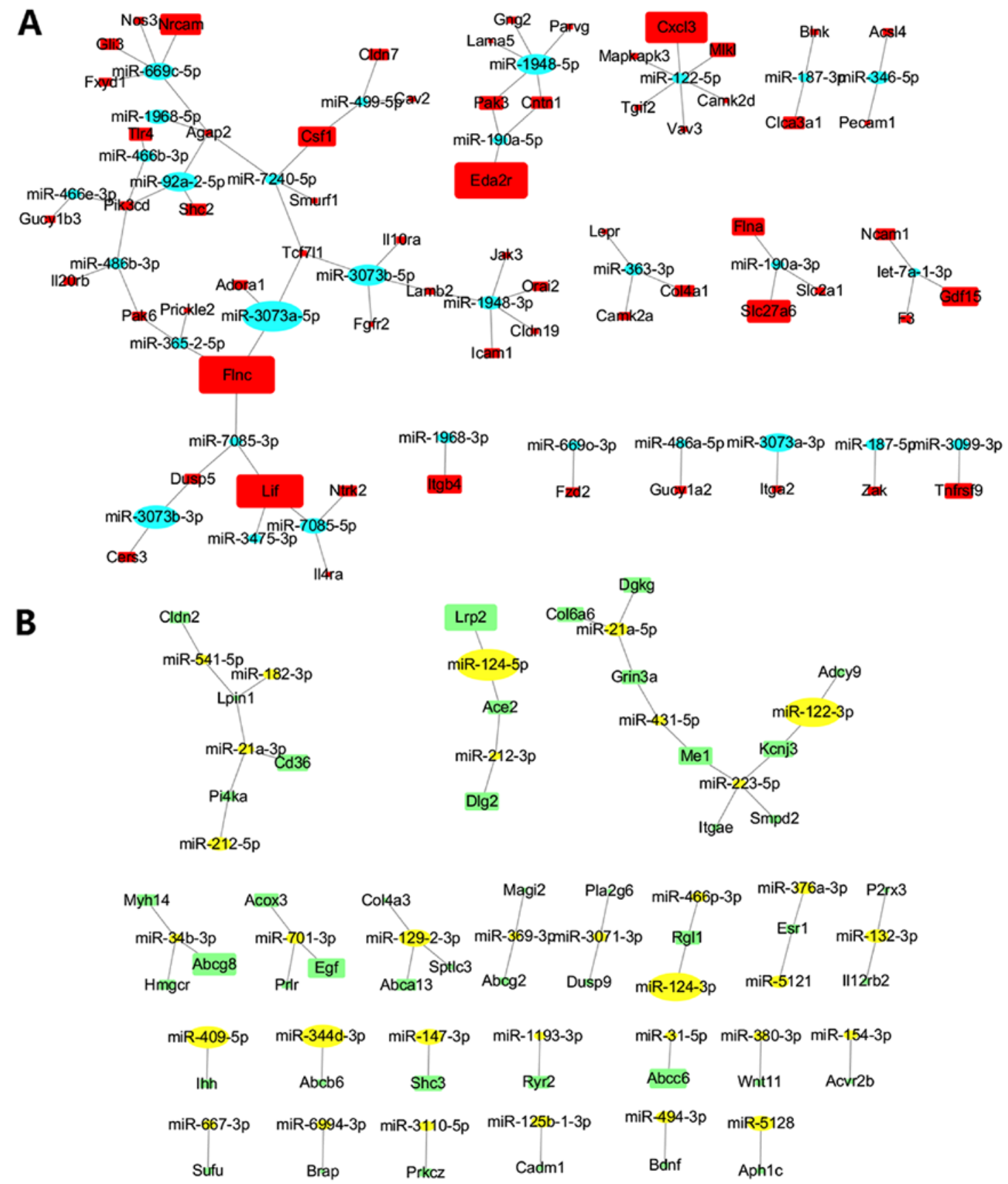

Figure 7. Networks of DE miRNAs and concurrently oppositely regulated DE mRNAs involved in signaling pathways and ECM-associated pathways following AA treatment. (A) Networks of decreased DE miRNAs and concurrently increased target DE mRNAs. (B) Networks of increased DE miRNAs and concurrently decreased target DE mRNAs. DE miRNAs are indicated by an oval. DE mRNAs are indicated by a rectangle. The increased DE miRNAs are marked in yellow and the decreased DE miRNAs are marked in blue. The increased DE mRNAs are marked in red and the decreased DE mRNAs are marked in green. The magnitude of the differential expression changes in DE miRNAs and DE mRNAs following AA treatment is indicated by the size of the node. AA, aristolochic acid; ECM, extracellular matrix; DE, differentially expressed; miRNA/miR, microRNA.

networks included 54 pairs of increased DE miRNAs and concurrent decreased DE mRNAs (Fig. 7B), and 80 pairs of decreased DE miRNAs and concurrent increased DE mRNAs (Fig. 7A). Among these 63 DE miRNAs, 44 miRNAs had $>1$ DE mRNA target. Among them, the increased DE miRNAs included the miR-21 family (miR-21a-5p and miR-21a-3p), miR-124 family (miR-124-5p and miR-124-3p), miR-129-2-3p, miR-34b-3p, and so on; the decreased DE miRNAs included the miR-3073 family (miR-3073a-5p, miR-3073a-3p and miR-3073b-5p), miR-1948 family (miR-1948-5p and miR-1948-3p), miR-122-5p, miR-669c-5p and let7a-1-3p (Fig. 7; Table SII).

\section{Discussion}

In the present study, short-term exposure (5 days) to aristolochic acid (AA) induced renal injury and tubulointerstitial fibrosis in acute aristolochic acid nephropathy (AAN) in mice. To the best of our knowledge, the present study is the first to report the changes in genome-wide DE miRNAs and DE mRNAs, and their regulatory pairs and networks in acute AAN.

There was notable dysregulation of the miRNA expression profile following treatment with AA. Specifically, 82 DE miRNAs were identified following AA treatment, the 
most notable alterations of which occurred in the profibrotic miR-21, miR-433 and miR-132 families, and the oncogenic miR-34 family, which were significantly increased, and in the anti-fibrotic miR-122-5p and let-7a-1-3p, which were significantly decreased. Similar increases in the miR-21 and miR-34 families following long-term exposure to a carcinogenic dose (10 mg/kg/day for 12 weeks) of AA in rats have been previously reported in rats $(25,26)$. Activity of the TGF- $\beta / \mathrm{Smad} 3$ signaling pathway is increased in renal fibrosis by miR-21 $(25,26)$ via Smad7, PTEN, miR-433 (27) and Azin1. Silencing of miR-132 was reported to reduce renal fibrosis $(28,29)$. Significant downregulation of let-7a was reported to be associated with renal fibrosis in chronic kidney disease (30).

Global mRNA profiling was notably altered following AA treatment. Amino acid metabolism, fatty acid metabolism, 'complement and coagulation cascades', 'ECM-receptor interaction' and 'focal adhesion' were the most significantly enriched pathways, based on KEGG analysis of DE mRNAs. The pathways of 'complement and coagulation cascades', 'ECM-receptor interaction' and 'focal adhesion' directly participate in the process of tubulointerstitial fibrosis $(23,31)$. Amino acid metabolism pathways are involved in the production of matrix collagen during fibrosis, which is enriched in glycine, hydroxyproline, and hydroxylysine, and other amino acid-derived compounds (32).

Additionally, GO and KEGG analysis were used to analyze the 624 DE mRNAs regulated by DE miRNAs. The renal tubular injury was largely the result of death or apoptosis of tubular epithelial cells $(33,34)$. The following tubulointerstitial fibrosis was induced by infiltration of immune cells, production and secretion of pro-fibrosis factors, downregulation and inhibition of anti-fibrosis factors, myofibroblasts activation, EMT, ECM production, tubular atrophy and others (7). In this process, the most altered biological process in $\mathrm{GO}$ analysis may serve an important role, which included 'biological regulation', 'metabolic process' and 'response to stimulus' in the biological process category, 'binding' in the molecular function category, and 'organelle' and 'membrane part' in the cellular component category. This process was also affected by the most altered pathways in KEGG analysis, which included 'signal transduction', metabolism, 'endocrine system', 'cellular community - eukaryotes' and 'immune system'. Additionally, 'cancers' were one of the top changed pathways, as AA is also a carcinogen, and EMT in fibrosis is an important pathway in cancer.

In KEGG, similar to the results obtained from analysis of the total DE miRNAs, ECM-associated pathways, metabolic pathways and cancer-associated pathways were the most enriched KEGG pathways. However, unlike the results of total DE miRNAs, signaling pathways were identified as the most enriched KEGG pathways regulated by DE miRNAs. A total of 40 signaling pathways were identified. In the most significantly altered signaling pathway in this study, fibrosis was reported in previous studies to be involved in the 'Hedgehog signaling pathway' (35-37), 'ABC transporters' (38), 'ErbB signaling pathway' $(39,40)$, 'cAMP signaling pathway' $(41,42)$, 'Relaxin signaling pathway' $(43,44)$, 'Ras signaling pathway' $(45,46)$ and 'PI3K-Akt signaling pathway' $(47,48)$. Additionally, six ECM-associated pathways were directly associated with renal fibrosis.
It has been suggested that DE miRNAs serve an important role in renal injury and tubulointerstitial fibrosis by regulating the DE mRNAs enriched in the signaling pathways and ECM-associated pathways. In the aforementioned 40 enriched signaling pathways and 6 ECM-associated pathways, the opposite direction regulatory networks of $63 \mathrm{DE}$ miRNAs and their 107 DE mRNA targets were drawn. A total of 134 opposite direction regulatory pairs of DE miRNAs and DE mRNAs were included in these regulatory networks. The majority of the 63 DE miRNAs had $>1$ DE mRNA target. For example, the increased DE miRNAs with the largest numbers of targets included the miR-21 family (miR-21a-5p and miR-21a-3p), the miR-124 family (miR-124-5p and miR-124-3p), miR-129-2-3p and miR-34b-3p. The decreased DE miRNAs with the largest numbers of targets included miR-122-5p, the miR-3073 family (miR-3073a-5p, miR-3073a-3p and miR-3073b-5p), the miR-1948 family (miR-1948-5p and miR-1948-3p), miR-669c-5p and let7a-1-3p. Among these, fibrosis was reported to be regulated by the increase in expression of the miR-21 family (49-54), miR-124 family $(55,56)$ and miR-34b-3p $(57,58)$, and the decrease in miR-122-5p (59-61) and let7a-1-3p (62). The present study was the first to report that AAN was associated with an increase in miR-129-2-3p expression and a decrease in the expression levels of the miR-3073 family (miR-3073a-5p, miR-3073a-3p and miR-3073b-5p), miR-1948 family (miR-1948-5p and miR-1948-3p) and miR-669c-5p.

In the present self-controlled study, mice were treated with AA, following unilateral nephrectomy. This self-control design may help increase the sensitivity and accuracy of results by decreasing the interference of individual variations. However, there may be other influencing factors, including anesthesia, hyper-perfusion and residual renal function following unilateral nephrectomy. These factors were likely less influencing than the AA-induced acute renal injury. Although these factors often had a notable effect under the background of hypertension, diabetes, genetic defect, toxic drugs or other harmful conditions, no significant changes were reported in the control mice group given only unilateral nephrectomy (63-65). The nature and severity of the renal response to unilateral nephrectomy were reported to be strain-dependent in mice (66). It has been reported that the unilateral nephrectomy did not induce sclerosis or fibrosis in the glomerulus, tubulointerstitium or vasculature after 8 weeks in the C57 strain mice (66).

Acute AAN in humans results in AA-induced acute kidney injury and may progress to chronic kidney disease (CKD, as necroinflammation, an auto amplification loop between tubular cell death/apoptosis and interstitial inflammation, results in continued renal injury and tubulointerstitial fibrosis (67-69). Halting the progression of CKD following AA exposure by inhibition or even resolution of AA-induced renal tubular injury and tubulointerstitial fibrosis should be considered as a potential therapeutic approach. The present study identified DE miRNAs, their mRNA targets and the enriched pathways of the mRNA targets, which may assist in improving the understanding of the underlying mechanisms. The DE miRNAs and their regulated mRNAs represent potential targets that may protect the kidney from continued tubular injury and tubulointerstitial fibrosis following AA exposure, and may assist in the development of novel clinical therapies for treatment of CKD following AA exposure. 
In conclusion, in the present self-controlled study, renal injury and tubulointerstitial fibrosis were induced by short-term treatment with AA in mice, and genome-wide DE miRNAs and DE mRNAs, and their regulated pairs and networks were identified. The present results may assist in improving the understanding of the role of the DE miRNAs and their mRNA targets in the pathophysiology of renal injury and tubulointerstitial fibrosis in acute AAN.

\section{Acknowledgements}

Not applicable.

\section{Funding}

The present study was supported by grants from Jiangsu Commission of Health (grant nos. F201439 and QNRC305) and Changzhou Commission of Health (grant no. QN201724).

\section{Availability of data and materials}

The datasets used and/or analyzed during the current study are available from the corresponding author on reasonable request.

\section{Authors' contributions}

XL designed this study. ZZ participated in the design and writing of the study, the major experiments and data analysis. $\mathrm{XX}, \mathrm{YS}$ and $\mathrm{YZ}$ participated in the in vivo experiments. XX, $\mathrm{WQ}$ and $\mathrm{FW}$ participated in the in vitro experiments. $\mathrm{XZ}$, $\mathrm{CB}, \mathrm{HH}$ and SL analyzed the data from the deep sequencing results of miRNA and mRNA. All authors read and approved the final manuscript.

\section{Ethics approval and consent to participate}

The present study was approved by the Ethics Committee of the Children's Hospital of Soochow University (approval no. 2020-CHSU-012).

\section{Patient consent for publication}

Not applicable.

\section{Competing interests}

The authors declare that they have no competing interests.

\section{References}

1. Schaneberg BT, Applequist WL and Khan IA: Determination of aristolochic acid I and II in North American species of Asarum and Aristolochia. Pharmazie 57: 686-689, 2002.

2. Michl J, Jennings HM, Kite GC, Ingrouille MJ, Simmonds MS and Heinrich M: Is aristolochic acid nephropathy a widespread problem in developing countries? A case study of Aristolochia indica L. in Bangladesh using an ethnobotanical-phytochemical approach. J Ethnopharmacol 149: 235-244, 2013.

3. Bara T Jr, Gurzu S, Sugimura H, Bara T, Beleaua MA and Jung I: A systematic review of the possible carcinogenic role of the aristolochic acid. Rom J Morphol Embryol 58: 41-44, 2017.
4. Michl J, Ingrouille MJ, Simmonds MSJ and Heinrich M: Naturally occurring aristolochic acid analogues and their toxicities. Nat Prod Rep 31: 676-693, 2014.

5. Yang L, Li X and Wang H: Possible mechanisms explaining the tendency towards interstitial fibrosis in aristolochic acid-induced acute tubular necrosis. Nephrol Dial Transplant 22: 445-456, 2007.

6. Jadot I, Decleves AE, Nortier J and Caron N: An integrated view of aristolochic acid nephropathy: Update of the literature. Int J Mol Sci 18: 297, 2017.

7. Humphreys BD: Mechanisms of renal fibrosis. Annu Rev Physiol 80: 309-326, 2018.

8. Rockey DC, Bell PD and Hill JA: Fibrosis-a common pathway to organ injury and failure. N Engl J Med 372: 1138-1149, 2015.

9. Yeh YC, Wei WC, Wang YK, Lin SC, Sung JM and Tang MJ: Transforming growth factor- $\{$ beta 1 induces Smad3-dependent \{beta\}1 integrin gene expression in epithelial-to-mesenchymal transition during chronic tubulointerstitial fibrosis. Am J Pathol 177: 1743-1754, 2010.

10. Yang Y, Feng XJ, Liu XY, Wang LH and Zheng GP: The effect of transforming growth factor $\beta(1)$ in the transition of bone marrow-derived macrophages into myofibroblasts during renal fibrosis. Zhonghua Nei Ke Za Zhi 56: 610-613, 2017 (In Chinese).

11. Gebert LFR and MacRae IJ: Regulation of microRNA function in animals. Nat Rev Mol Cell Biol 20: 21-37, 2019.

12. Bushati N and Cohen SM: MicroRNA functions. Annu Rev Cell Dev Biol 23: 175-205, 2007.

13. Chung AC and Lan HY: MicroRNAs in renal fibrosis. Front Physiol 6: 50, 2015.

14. Wang B and Ricardo S: Role of microRNA machinery in kidney fibrosis. Clin Exp Pharmacol Physiol 41: 543-550, 2014.

15. Tang C, Xie Y and Yan W: 1 AASRA: An anchor alignment-based small RNA. bioRxiv 2017 (Epub ahead for print).

16. Nawrocki EP and Eddy SR: Infernal 1.1: 100-fold faster RNA homology searches. Bioinformatics 29: 2933-2935, 2013.

17. Kim D, Langmead B and Salzberg SL: HISAT: A fast spliced aligner with low memory requirements. Nat Methods 12: 357-360, 2015

18. Kruger J and Rehmsmeier M: RNAhybrid: microRNA target prediction easy, fast and flexible. Nucleic Acids Res 34(Web Server Issue): W451-W454, 2006.

19. John B, Enright AJ, Aravin A, Tuschl T, Sander C and Marks DS: Human microRNA targets. PLoS Biol 2: e363, 2004.

20. Mi H, Muruganujan A, Ebert D, Huang $X$ and Thomas PD: PANTHER version 14: More genomes, a new PANTHER GO-slim and improvements in enrichment analysis tools. Nucleic Acids Res 47D: D419-D426, 2019.

21. Kanehisa M, Furumichi M, Tanabe M, Sato Y and Morishima K: KEGG: New perspectives on genomes, pathways, diseases and drugs. Nucleic Acids Res 45D: D353-D361, 2017.

22. Livak KJ and Schmittgen TD: Analysis of relative gene expression data using real-time quantitative PCR and the 2(-Delta Delta C(T)) method. Methods 25: 402-408, 2001.

23. Horowitz JC and Thannickal VJ: Mechanisms for the resolution of organ fibrosis. Physiology (Bethesda) 34: 43-55, 2019.

24. Schafer S, Viswanathan S, Widjaja AA, Lim WW, Moreno-Moral A, DeLaughter DM, Ng B, Patone G, Chow K, Khin E, et al: IL-11 is a crucial determinant of cardiovascular fibrosis. Nature 552: 110-115, 2017.

25. Li Z, Qin T, Wang K, Hackenberg M, Yan J, Gao Y, Yu LR, Shi L, $\mathrm{Su} \mathrm{Z}$ and Chen $\mathrm{T}$ : Integrated microRNA, mRNA, and protein expression profiling reveals microRNA regulatory networks in rat kidney treated with a carcinogenic dose of aristolochic acid. BMC Genomics 16: 365, 2015.

26. Meng F, Li Z, Yan J, Manjanatha M, Shelton S, Yarborough S and Chen T: Tissue-specific microRNA responses in rats treated with mutagenic and carcinogenic doses of aristolochic acid. Mutagenesis 29: 357-365, 2014

27. Li R, Chung AC, Dong Y, Yang W, Zhong X and Lan HY: The microRNA miR-433 promotes renal fibrosis by amplifying the TGF- $\beta /$ Smad3-Azin1 pathway. Kidney Int 84: 1129-1144, 2013.

28. Bijkerk R, de Bruin RG, van Solingen C, van Gils JM, Duijs JM, van der Veer EP, Rabelink TJ, Humphreys BD and van Zonneveld AJ: Silencing of microRNA-132 reduces renal fibrosis by selectively inhibiting myofibroblast proliferation. Kidney Int 89: 1268-1280, 2016.

29. Zhou SG, Zhang W, Ma HJ, Guo ZY and Xu Y: Silencing of IncRNA TCONS_00088786 reduces renal fibrosis through miR-132. Eur Rev Med Pharmacol Sci 22: 166-173, 2018. 
30. Muralidharan J, Ramezani A, Hubal M, Knoblach S, Shrivastav S, Karandish S, Scott R, Maxwell N, Ozturk S, Beddhu S, et al: Extracellular microRNA signature in chronic kidney disease. Am J Physiol Renal Physiol 312: F982-F991, 2017.

31. Liu Y: Cellular and molecular mechanisms of renal fibrosis. Nat Rev Nephrol 7: 684-696, 2011.

32. Gaugg MT, Engler A, Bregy L, Nussbaumer-Ochsner Y, Eiffert L, Bruderer T, Zenobi R, Sinues P and Kohler M: Molecular breath analysis supports altered amino acid metabolism in idiopathic pulmonary fibrosis. Respirology 24: 437-444, 2019.

33. Park JS, Choi HI, Kim DH, Kim CS, Bae EH, Ma SK and Kim SW: Alpha-lipoic acid attenuates p-cresyl sulfate-induced renal tubular injury through suppression of apoptosis and autophagy in human proximal tubular epithelial cells. Biomed Pharmacother 112: 108679, 2019.

34. Dong Q, Jie Y, Ma J, Li C, Xin T and Yang D: Renal tubular cell death and inflammation response are regulated by the MAPK-ERK-CREB signaling pathway under hypoxia-reoxygenation injury. J Recept Signal Transduct Res 39: 383-391, 2019

35. Kramann R: Hedgehog Gli signalling in kidney fibrosis. Nephrol Dial Transplant 31: 1989-1995, 2016.

36. Zhou D, Tan RJ and Liu Y: Sonic hedgehog signaling in kidney fibrosis: A master communicator. Sci China Life Sci 59: 920-929, 2016.

37. Fabian SL, Penchev RR, St-Jacques B, Rao AN, Sipila P, West KA, McMahon AP and Humphreys BD: Hedgehog-Gli pathway activation during kidney fibrosis. Am J Pathol 180: 1441-1453, 2012.

38. Kai Y, Yoneyama H, Koyama J, Hamada K, Kimura H and Matsushima K: Treatment with chondroitinase ABC alleviates bleomycin-induced pulmonary fibrosis. Med Mol Morphol 40: 128-140, 2007.

39. Vermeulen Z, Hervent AS, Dugaucquier L, Vandekerckhove L, Rombouts M, Beyens M, Schrijvers DM, De Meyer GRY, Maudsley S, De Keulenaer GW and Segers VFM: Inhibitory actions of the NRG-1/ErbB4 pathway in macrophages during tissue fibrosis in the heart, skin, and lung. Am J Physiol Heart Circ Physiol 313: H934-H945, 2017.

40. Scheving LA,Zhang X, Threadgill DW and Russell WE: Hepatocyte ERBB3 and EGFR are required for maximal CCl4-induced liver fibrosis. Am J Physiol Gastrointest Liver Physiol 311: G807-G816, 2016.

41. Essam RM, Ahmed LA, Abdelsalam RM and El-Khatib AS Phosphodiestrase-1 and 4 inhibitors ameliorate liver fibrosis in rats: Modulation of cAMP/CREB/TLR4 inflammatory and fibrogenic pathways. Life Sci 222: 245-254, 2019.

42. Han K, Zhang Y and Yang Z: Cilostazol protects rats against alcohol-induced hepatic fibrosis via suppression of TGF- $\beta 1 /$ CTGF activation and the cAMP/Epacl pathway. Exp Ther Med 17: 2381-2388, 2019.

43. Kanai AJ, Konieczko EM, Bennett RG, Samuel CS and Royce SG: Relaxin and fibrosis: Emerging targets, challenges, and future directions. Mol Cell Endocrinol 487: 66-74, 2019.

44. Zheng G, Cai J, Chen X, Chen L, Ge W, Zhou X and Zhou H: Relaxin ameliorates renal fibrosis and expression of endothelial cell transition markers in rats of isoproterenol-induced heart failure. Biol Pharm Bull 40: 960-966, 2017.

45. Newbury LJ, Wang JH, Hung G, Hendry BM and Sharpe CC: Inhibition of Kirsten-Ras reduces fibrosis and protects against renal dysfunction in a mouse model of chronic folic acid nephropathy. Sci Rep 9: 14010,2019.

46. Zhou G, Li J, Zeng T, Yang P and Li A: The regulation effect of WNT-RAS signaling in hypothalamic paraventricular nucleus on renal fibrosis. J Nephrol 33: 289-297, 2020

47. Wang J, Zhu H, Huang L, Zhu X, Sha J, Li G, Ma G, Zhang W, Gu M and Guo Y: Nrf2 signaling attenuates epithelial-to-mesenchymal transition and renal interstitial fibrosis via PI3K/Akt signaling pathways. Exp Mol Pathol 111: 104296, 2019.

48. Dou F, Liu Y, Liu L, Wang J, Sun T, Mu F, Guo Q, Guo C, Jia N, Liu W, et al: Aloe-Emodin ameliorates renal fibrosis via inhibiting PI3K/Akt/mTOR signaling pathway in vivo and in vitro. Rejuvenation Res 22: 218-229, 2019.

49. Sun L, Xu T, Chen Y, Qu W, Sun D, Song X, Yuan Q and Yao L: Pioglitazone attenuates kidney fibrosis via miR-21-5p modulation. Life Sci 232: 116609, 2019.

50. Tian C, Wang Y, Chang H, Li J and La X: Spleen-kidney supplementing formula alleviates renal fibrosis in diabetic rats via TGF- $\beta 1$-miR-21-PTEN signaling pathway. Evid Based Complement Alternat Med 2018: 3824357, 2018
51. Tang CR, Luo SG, Lin X, Wang J and Liu Y: Silenced miR-21 inhibits renal interstitial fibrosis via targeting ERK1/2 signaling pathway in mice. Eur Rev Med Pharmacol Sci 23 (3 Suppl): S110-S116, 2019

52. McClelland AD, Herman-Edelstein M, Komers R, Jha JC Winbanks CE, Hagiwara S, Gregorevic P, Kantharidis P and Cooper ME: miR-21 promotes renal fibrosis in diabetic nephropathy by targeting PTEN and SMAD7. Clin Sci (Lond) 129: $1237-1249,2015$

53. Wang J, Gao Y, Ma M, Li M, Zou D, Yang J, Zhu Z and Zhao X Effect of miR-21 on renal fibrosis by regulating MMP-9 and TIMP1 in kk-ay diabetic nephropathy mice. Cell Biochem Biophys 67: 537-546, 2013.

54. Zhong X, Chung AC, Chen HY, Meng XM and Lan HY: Smad3-mediated upregulation of miR-21 promotes renal fibrosis. J Am Soc Nephrol 22: 1668-1681, 2011.

55. Zhou H, Qiu ZZ, Yu ZH, Gao L, He JM, Zhang ZW and Zheng J: Paeonol reverses promoting effect of the HOTAIR/miR-124/Notch1 axis on renal interstitial fibrosis in a rat model. J Cell Physiol 234: 14351-14363, 2019.

56. Zhou H, Gao L, Yu ZH, Hong SJ, Zhang ZW and Qiu ZZ: lncRNA HOTAIR promotes renal interstitial fibrosis by regulating Notch1 pathway via the modulation of miR-124. Nephrology (Carlton) 24: 472-480, 2019

57. Disayabutr S, Kim EK, Cha SI, Green G, Naikawadi RP, Jones KD, Golden JA, Schroeder A, Matthay MA, Kukreja J, et al: miR-34 miRNAs regulate cellular senescence in type II alveolar epithelial cells of patients with idiopathic pulmonary fibrosis. PLoS One 11: e158367, 2016.

58. Li WQ, Chen C, Xu MD, Guo J, Li YM, Xia QM, Liu HM, He J, Yu HY and Zhu L: The rno-miR-34 family is upregulated and targets ACSL1 in dimethylnitrosamine-induced hepatic fibrosis in rats. FEBS J 278: 1522-1532, 2011.

59. Sun Y, Wang H, Li Y, Liu S, Chen J and Ying H: miR-24 and miR-122 negatively regulate the transforming growth Factor- $\beta / \mathrm{smad}$ signaling pathway in skeletal muscle fibrosis. Mol Ther Nucleic Acids 11: 528-537, 2018.

60. Lou G, Yang Y, Liu F, Ye B, Chen Z, Zheng M and Liu Y: miR-122 modification enhances the therapeutic efficacy of adipose tissue-derived mesenchymal stem cells against liver fibrosis. J Cell Mol Med 21: 2963-2973, 2017.

61. Halasz T, Horvath G, Par G, Werling K, Kiss A, Schaff Z and Lendvai G: miR-122 negatively correlates with liver fibrosis as detected by histology and FibroScan. World J Gastroenterol 21: 7814-7823, 2015.

62. Li N, Wang LJ, Xu WL, Liu S and Yu JY: MicroRNA3795p suppresses renal fibrosis by regulating the LIN28/let7 axis in diabetic nephropathy. Int J Mol Med 44: 1619-1628, 2019.

63. Cevikbas F, Schaefer L, Uhlig P, Robenek H, Theilmeier G, Echtermeyer F and Bruckner P: Unilateral nephrectomy leads to up-regulation of syndecan-2- and TGF-beta-mediated glomerulosclerosis in syndecan-4 deficient male mice. Matrix Biol 27: 42-52, 2008.

64. Xia Y, Jin X, Yan J, Entman ML and Wang Y: CXCR6 plays a critical role in angiotensin II-induced renal injury and fibrosis. Arterioscler Thromb Vasc Biol 34: 1422-1428, 2014.

65. Uil M, Scantlebery AML, Butter LM, Larsen PWB, de Boer OJ, Leemans JC, Florquin S and Roelofs JJTH: Combining streptozotocin and unilateral nephrectomy is an effective method for inducing experimental diabetic nephropathy in the 'resistant' C57Bl/6J mouse strain. Sci Rep 8: 5542, 2018.

66. Esposito C, He CJ, Striker GE, Zalups RK and Striker LJ: Nature and severity of the glomerular response to nephron reduction is strain-dependent in mice. Am J Pathol 154: 891-897, 1999.

67. Liu BC, Tang TT and Lv LL: How tubular epithelial cell injury contributes to renal fibrosis. Adv Exp Med Biol 1165: 233-252, 2019.

68. Liu BC, Tang TT, Lv LL and Lan HY: Renal tubule injury: A driving force toward chronic kidney disease. Kidney Int 93: 568-579, 2018

69. Youl ENH, Husson C, El Khattabi C, El Mere S, Decleves AE, Pochet S, Nortier J and Antoine MH: Characterization of cytotoxic effects of aristolochic acids on the vascular endothelium. Toxicol In Vitro 65: 104811, 2020.

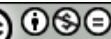

This work is licensed under a Creative Commons Attribution-NonCommercial-NoDerivatives 4.0 International (CC BY-NC-ND 4.0) License. 